\title{
Renewal of extinguished instrumental responses: independence from Pavlovian processes and dependence on outcome value
}

\author{
Sabrina R. Cohen-Hatton • R. C. Honey
}

Published online: 3 July 2013

(C) Psychonomic Society, Inc. 2013

\begin{abstract}
The source of renewal of instrumental responding in rats was investigated. In Experiment 1, two responses (R1 and R2) were reinforced with one outcome (O1) in contexts $\mathrm{A}$ and $\mathrm{B}$ (i.e., $\mathrm{R} 1 \rightarrow \mathrm{O} 1, \mathrm{R} 2 \rightarrow \mathrm{O} 1$ ), and then $\mathrm{R} 2$ was extinguished in A and R1 was extinguished in B. At test, the rate of R1 was higher than that of R2 in context A, and the reverse was the case in context $\mathrm{B}$ : Renewed responding was independent of the Pavlovian context $\rightarrow \mathrm{O} 1$ associations. In Experiment 2, all rats received $\mathrm{R} 1 \rightarrow \mathrm{O} 1$ and $\mathrm{R} 2 \rightarrow \mathrm{O} 2$ trials in $\mathrm{A}$ and then were placed in $\mathrm{B}$, where they were sated on $\mathrm{O} 2$ and either did (Group Extinction) or did not (Group No Extinction) receive concurrent extinction of $\mathrm{R} 1$ and $\mathrm{R} 2$. At test, we found more responding in A than in B for Group Extinction, but not for Group No Extinction, and the renewed responding in A was as sensitive to the current value of the outcome as responding that had not been subject to extinction (i.e., the rate was higher for R1 than for R2). That is, the renewed responding was goaldirected. These results identify the removal of contextual inhibion of either the response or the response $\rightarrow$ outcome associaon as potenal bases for renewal, and the response $\rightarrow$ outcome associaon as the source of renewed responding.
\end{abstract}

Keywords Instrumental conditioning · Extinction ·

Goal-directed behavior $\cdot$ Renewal

The experimental context in which conditioning and extinction occur exerts a profound influence over the conditioned behavior. One well-documented example of this influence is the ABA renewal effect, wherein a response that has been

S. R. Cohen-Hatton • R. C. Honey $(\bowtie)$

School of Psychology, Cardiff University, Tower Building,

Park Place, Cardiff CF10 3AT, UK

e-mail: Honey@cardiff.ac.uk conditioned in one context (A), and then extinguished in another context (B), returns when it is assessed in context A. The overwhelming majority of the examples of this effect involve Pavlovian conditioning, in which the response in question is one that had been conditioned (and then extinguished) to a conditioned stimulus (CS) presented within the contexts (A and B; Bouton \& Bolles, 1979; for a review, see Bouton, 2004). However, analogous effects have also been observed using instrumental conditioning procedures. For example, lever pressing established in context $\mathrm{A}$ and extinguished in context $\mathrm{B}$ is observed again when it is assessed in context $\mathrm{A}$ (e.g., Bouton, Todd, Vurbic, \& Winterbauer, 2011; Nakajima, Tanaka, Urushihara, \& Imada, 2000; Nakajima, Urushihara, \& Masaki, 2002; Todd, Winterbauer, \& Bouton, 2012). Instrumental renewal effects have also been demonstrated in which drugs of misuse serve as the reinforcer (e.g., Bossert, Liu, Lu, \& Shaham, 2004; Crombag \& Shaham, 2002; Hamlin, Clemens, \& McNally, 2008). Although the origin of Pavlovian renewal effects has been examined in some detail (e.g., Bouton \& King, 1983; Bouton \& Swartzentruber, 1986; Rescorla, 2008), the equivalent effect in instrumental conditioning has been the subject of less detailed analysis.

One obvious way in which contexts might exert control over instrumental responding is through the Pavlovian properties that they acquire when paired with reinforcement (during training) and nonreinforcement (during extinction). Consider the case of ABA renewal. The first stage of instrumental conditioning provides the necessary conditions for the context (e.g., A) to acquire an excitatory association with the outcome, and the second, extinction stage provides the conditions that should allow context B to gain an inhibitory association with the same outcome. These Pavlovian associations might well contribute to the renewal of instrumental responding. It is well-established that Pavlovian CSs can 
modulate instrumental performance from studies of Pavlovianto-instrumental transfer (PIT; for a review, see Holmes, Marchand, \& Coutureau, 2010). Although the origin of (outcome-selective) PIT is contentious (see Cohen-Hatton, Haddon, George, \& Honey, 2013), it is certainly the case that a context paired with an outcome can augment instrumental responding (Pearce \& Hall, 1979; see also Baker, Steinwald, \& Bouton, 1991; Colwill \& Rescorla, 1988; Rescorla \& Solomon, 1967). The suggestion that instrumental renewal effects are a direct product of the excitatory Pavlovian properties of the training context is undermined, although not precluded, by several observations. The effect can be observed when testing occurs in a context (C) that one could argue is likely to be associatively neutral (in $\mathrm{ABC}$ renewal), and $\mathrm{ABA}$ renewal is relatively immune to extinction of context $\mathrm{A}$ before the test (see Bouton et al., 2011). The latter observations do not, however, exclude the possibility that the extinction context, B, might have gained inhibitory properties, the removal of which increases responding in context $\mathrm{C}$. That is, context $\mathrm{B}$ might have come to inhibit the memory of the outcome that was absent when the response was not reinforced during extinction, and this inhibitory association could affect the vigor of instrumental responding in context B but not context C (cf. Delamater, LoLordo, \& Sosa, 2003). To rule out explanations of instrumental renewal that are based on the (inhibitory or excitatory) Pavlovian properties of the contexts, a procedure is required that equates these properties within a renewal design.

The principal aim of Experiment 1 was to assess whether a renewal effect could be observed under conditions in which the Pavlovian properties of the contexts (i.e., A and B) were equated. The design was modeled on one employed by Rescorla (2008; see also Harris, Jones, Bailey, and Westbrook, 2000) to address the equivalent issue in the Pavlovian ABA renewal procedure. This design produced a renewal effect in the Pavlovian case, and if the same were true in the instrumental case, this would constrain the range of explanations of instrumental renewal that remain in play. For example, in the case of the renewal of Pavlovian conditioning, contexts have often been described as acting in a hierarchical or occasionsetting fashion, on the link or links between the CS and the outcome or the absence of the outcome (see Bouton \& Swartzentruber, 1986; Honey \& Watt, 1999). The application of such a hierarchical analysis to the contextual control of instrumental responding assumes that the contexts are acting on the link between the response and the outcome (see Rescorla, 1991), and it is worth remembering that Skinner (1938) referred to discriminative stimuli as setting the occasion for instrumental responding. Set within an associative analysis of instrumental conditioning, ABA renewal could be based on the test context (A) gating the excitatory response $\rightarrow$ outcome link or on removing the context B's gating of the inhibitory response $\rightarrow$ outcome link. The finding that a renewal effect can be observed when conditioning and extinction occur in context A and testing occurs in B is certainly consistent with the idea that the extinction context gates the inhibitory response $\rightarrow$ outcome link (Bouton, Winterbauer, \& Todd, 2012).

Some recent evidence is already showing that renewed instrumental responding can be observed under conditions in which the reinforcement histories of the contexts are equated, thus ruling out the possibility that differential contextoutcome associations are the sole mechanism for renewal (Todd, in press; see also Nakajima et al., 2002). Even when renewed instrumental responding is observed under conditions in which the influence of the Pavlovian associations with the outcome could be excluded, this does not necessarily suggest that renewed responding originates in a response $\rightarrow$ outcome association. It is also possible that renewed instrumental responding reflects a direct excitatory effect of the conditioning context (A) on the response at test or, conversely, the release from an inhibitory effect of the extinction context (e.g., B) on the response. That is, renewed responding might be a product of either excitatory or inhibitory context $\rightarrow$ response associations, rather than being dependent on response $\rightarrow$ outcome associations. The source of renewed instrumental responding has not been investigated, and a secondary aim of Experiment 1 - and the primary aim of Experiment 2-was to do this. The approach that we adopted was to examine whether the current value of the outcome affected renewed responding (Adams, 1982; Adams \& Dickinson, 1981). If renewed responding was not affected by the value of the outcome, this would indicate that the contexts were exerting a direct effect on the response motor program, but if renewed responding was sensitive to the value of the outcome, this would implicate response $\rightarrow$ outcome associations as a source of renewed responding. This issue is not just of theoretical significance. It has been argued that renewal effects have relevance to our understanding of the relapse effects observed following extinction treatments in, for example, drug-seeking behavior (Bouton, 2002, 2012; de Wit \& Dickinson, 2009; Laborda, McConnell, \& Miller, 2011). In the context of instrumental conditioning, which could be aligned with drug-seeking behavior, the nature of renewed responding has distinct relevance to its use as a model of relapse. Is renewed responding (relapse) based on a response $\rightarrow$ outcome association (and goal-directed), or is it independent of the outcome that engendered the original instrumental performance (and habitual)? The main purpose of Experiment 2 was to assess whether or not renewed instrumental responding is goal-directed.

\section{Experiment 1}

The design of Experiment 1 is summarized in the upper panel of Table 1. Rats first received training in which pressing the left and right levers (R1 and R2) resulted in the delivery of 
Table 1 Design of Experiments 1 and 2

\begin{tabular}{|c|c|c|c|}
\hline Instrumental & Extinction & Satiation & Test \\
\hline \multicolumn{4}{|l|}{ Experiment 1} \\
\hline $\mathrm{A}: \mathrm{R} 1 \rightarrow \mathrm{O} 1, \mathrm{R} 2 \rightarrow \mathrm{O} 1$ & A: R2- & $\mathrm{C}: \mathrm{O} 1$ or $\mathrm{O} 2$ & A: R1 vs. R2 \\
\hline$\&$ & $\&$ & & or \\
\hline $\mathrm{B}: \mathrm{R} 1 \rightarrow \mathrm{O} 1, \mathrm{R} 2 \rightarrow \mathrm{O} 1$ & B: R1- & & B: R1 vs. R2 \\
\hline \multicolumn{4}{|l|}{ Experiment 2} \\
\hline Group Extinction & \multicolumn{3}{|c|}{ Satiation/Extinction } \\
\hline $\mathrm{A}: \mathrm{R} 1 \rightarrow \mathrm{O} 1$ & \multirow{3}{*}{\multicolumn{2}{|c|}{$\mathrm{B}: \mathrm{O} 1 \ldots \mathrm{R} 1-/ \mathrm{R} 2-$}} & A: R1 vs. R2 \\
\hline$\&$ & & & or \\
\hline $\mathrm{A}: \mathrm{R} 2 \rightarrow \mathrm{O} 2$ & & & B: R1 vs. R2 \\
\hline \multicolumn{4}{|l|}{ Group No Extinction } \\
\hline $\mathrm{A}: \mathrm{R} 1 \rightarrow \mathrm{O} 1$ & \multirow{3}{*}{\multicolumn{2}{|c|}{ B: O1 ... }} & A: R1 vs. R2 \\
\hline$\&$ & & & or \\
\hline $\mathrm{A}: \mathrm{R} 2 \rightarrow \mathrm{O} 2$ & & & B: R1 vs. R2 \\
\hline
\end{tabular}

$\mathrm{R} 1$ and $\mathrm{R} 2$ refer to left and right lever presses; $\mathrm{O} 1$ and $\mathrm{O} 2$ denote food pellets and sucrose; - denotes no outcome; and A, B, and C are contexts

the same outcome (O1; e.g., a food pellet) in two contexts (A and B). After this, they were given access to one of the levers (supporting R1) in context B, and to the other lever (supporting R2) in context A, and lever pressing was not reinforced. The levers were introduced and retracted repeatedly during these sessions. This procedure allowed us to assess lever pressing and magazine entries separately during the two extinction sessions, and thereby to establish the extent to which any decline in the rate of lever pressing was a product of an increase in the tendency of rats to enter the magazine (e.g., as a product of extinction). Aside from this, the use of this discrete-trial procedure, like a free-operant procedure, allowed both the response and the cues associated with that response (e.g., the lever, and its spatial location) to undergo extinction. Finally, rats received a test in which both levers were presented in either context A or B, and the levels of R1 and $\mathrm{R} 2$ were assessed. Renewal would be evident if the rate of $\mathrm{R} 1$ was higher than the rate of $\mathrm{R} 2$ in context $\mathrm{A}$, and the reverse was the case in context $\mathrm{B}$. That is, responding should be more evident when the context in which the extinction of R1 and R2 was assessed was different from the context in which extinction had occurred ( $\mathrm{R} 1$ in context $\mathrm{A}$ and $\mathrm{R} 2$ in $\mathrm{B}$ ) than when the context was the same (R1 in B and R2 in A). It should be noted that the fact that both responses had been reinforced in contexts A and B means that any renewal effect must reflect something that had been learned during the extinction stage, as opposed to the retrieval of what had been acquired during conditioning (cf. Todd, in press).

We also undertook a preliminary assessment of whether or not any renewed responding was sensitive to the current value of the outcome. To do so, rats were sated with either $\mathrm{O} 1$ or a novel outcome $(\mathrm{O} 2)$ in a third context $(\mathrm{C})$ prior to the tests in contexts $\mathrm{A}$ and $\mathrm{B}$. If presentation of $\mathrm{O} 1$ resulted in a selective satiation effect, renewed responding should then be less evident after this treatment than after sating rats with $\mathrm{O} 2$. Although we recognize that this manipulation has some limitations (notably, it confounds value with the familiarity of the outcomes, an issue that Experiment 2 would avoid), Experiment 1 should still allow a renewal effect to be observed that would be independent of the Pavlovian properties of contexts $\mathrm{A}$ and $\mathrm{B}$ and dependent upon what was learnt during extinction: This was the primary goal of Experiment 1.

\section{Method}

Subjects Sixteen naïve Lister hooded rats were housed in pairs in standard rat cages and maintained on a $12-\mathrm{h} / 12-\mathrm{h}$ light/dark cycle (lights on at 7 a.m.). They had unrestricted access to water in their home cages and were maintained at $85 \%$ of their ad-lib weights (range: $300-325 \mathrm{~g}$ ) by giving them restricted access to food at the end of each day.

Apparatus All experimental sessions occurred within 16 identical conditioning boxes $(\mathrm{H} \times \mathrm{W} \times \mathrm{D}: 30 \mathrm{~cm} \times 24 \mathrm{~cm} \times$ $21 \mathrm{~cm}$; Med Associates, Georgia, VT) enclosed in soundattenuating chambers. These boxes consisted of aluminum front and back walls, clear acrylic sides and top, and a floor consisting of 0.48 -cm-diameter stainless steel rods spaced $1.6 \mathrm{~cm}$ apart, situated above a stainless steel tray. The walls and ceiling were lined with transparent Perspex behind which "wallpapers" were fixed. Four of the chambers had wallpapers consisting of black and white checks, four had white wallpaper with black spots, four had plain white wallpaper, and the remaining four had plain black wallpaper. Food pellets (45 mg; supplied by MLab, Richmond, IN) and sucrose solution $(15 \% \mathrm{w} / \mathrm{w})$ from a dipper $(0.1-\mathrm{ml}$ volume) could be separately delivered to a recessed food well that was equipped with infrared detectors was located in the center of the left wall. A ventilation fan maintained background noise (at $68 \mathrm{~dB}$ ). Two retractable levers, located $3 \mathrm{~cm}$ to the left and right of the food well, could be inserted into the box. A computerized interface (Med-PC) was used to insert the levers and to record food well entries and lever presses.

Procedure The rats were first trained to retrieve food pellets and sucrose from the food well in two separate 30-min sessions in a chamber with no wallpaper. Half of the rats in each group were trained to collect sucrose in the first session and pellets in the second, and the remainder received the reverse arrangement. The reinforcers were delivered on a random-time (RT) 60-s schedule; each second, food would become available with a 1 -in- 60 probability, after which the first food well entry would be rewarded.

Rats then received two types of instrumental conditioning trials (i.e., $\mathrm{R} 1 \rightarrow \mathrm{O} 1$ and $\mathrm{R} 2 \rightarrow \mathrm{O} 1$ ) that occurred in separate, alternating sessions in both contexts $\mathrm{A}$ and $\mathrm{B}$. For half of the 
rats, contexts $\mathrm{A}$ and $\mathrm{B}$ were boxes with spotted or checked wallpaper, and context $\mathrm{C}$ was either a box with black or white wallpaper; and for the remaining rats, contexts $\mathrm{A}$ and $\mathrm{B}$ were boxes with either black or white wallpaper, and context $\mathrm{C}$ was either a box with spotted or squared wallpaper. The identities of the boxes that served as A and B and the identity of the box that served as $\mathrm{C}$ were counterbalanced within the two subgroups described above. In these sessions, one lever was inserted, and pressing this lever (e.g., R1; pressing the left lever) resulted in the delivery of the designated outcome (O1; for half of the rats, $\mathrm{O} 1$ was food pellets, and for the remainder, it was sucrose). The rats first received four 15-min sessions in which each response (R1 and R2) was separately reinforced on a continuous reinforcement (CRF) schedule in each context in a counterbalanced order. Following CRF training, rats received a further 12 sessions of instrumental conditioning over 6 days, each lasting for $24 \mathrm{~min}$. These sessions were conducted at the same time of day for each rat, and reinforcers were delivered on a random-interval (RI) 30-s schedule with only one of the manipulanda being present in each session. Rats received one $\mathrm{R} 1 \rightarrow \mathrm{O} 1$ session and one $\mathrm{R} 2 \rightarrow \mathrm{O} 1$ session per day. These sessions were conducted in contexts $\mathrm{A}$ and $\mathrm{B}$ on alternating days. The alternating sequence of $\mathrm{R} 1 \rightarrow \mathrm{O} 1$ and $\mathrm{R} 2 \rightarrow \mathrm{O} 1$ training was counterbalanced, as were the orders in which rats were placed in contexts $\mathrm{A}$ and $\mathrm{B}$. The rate of lever pressing (in responses per minute, rpm) was used to assess instrumental conditioning.

Following instrumental training, on a single day, all of the rats received two 60-min extinction sessions, a satiation treatment, and a single test session. During the extinction sessions, the rats were placed in context $\mathrm{A}$, where the lever corresponding to R2 was inserted and responses were not followed by reinforcement (food or sucrose), and likewise were placed in context $\mathrm{B}$, where the lever corresponding to $\mathrm{R} 1$ was inserted and responses were not followed by reinforcement (food or sucrose). In these two sessions, the levers corresponding to R1 and R2 were inserted for a 10-s period prior to being retracted again. The lever was presented 120 times with a mean intertrial interval of $30 \mathrm{~s}$. The order in which these sessions occurred was counterbalanced, and the sessions were separated by $5 \mathrm{~min}$. Following these extinction treatments, rats were place in a novel context $(\mathrm{C})$, where half of them were sated on $\mathrm{O} 1$ and the remainder were sated on a novel outcome, O2. Rats received 120 presentations of the reinforcer on an RT-30 schedule. Approximately $5 \mathrm{~min}$ after this session, the rats received a single 5-min test session in either context A or context B. Both levers were present, and no reinforcers were delivered.

\section{Results}

As expected, lever pressing increased from the first day of RI 30 -s training $($ mean $=4.37 \mathrm{rpm})$ to the final, sixth day of training $($ mean $=9.17 \mathrm{rpm}), F(5,75)=14.22, M S E=4.82$, $p<.001$. The mean rates of lever pressing and magazine entries across the 12 blocks of ten extinction trials are shown in Fig. 1 (pooled across the various counterbalanced factors). An analysis of variance (ANOVA) confirmed the impression of a marked reduction across the extinction sessions in the rates of both lever pressing, $F(11,165)=20.89, M S E=2.94$, $p<.001$, and magazine entries, $F(11,165)=15.92, M S E=3.33$, $p<.001$. The results of principal interest from the test in Experiment 1 are summarized in Fig. 2, pooled across the various counterbalanced factors. Inspection of this figure shows that the rates of lever pressing on the levers that were tested in the same context as extinction (R2 in A and R1 in B) were lower than the rates on the levers that were tested in a different context (R1 in A and R2 in B). We also observed a numerical tendency for the rate of lever pressing to be lower when rats had been sated with the training reinforcer, O1, than when they had been sated with the novel reinforcer, O2. An ANOVA with Test Context (same or different) as a withinsubjects factor and Devaluation $(\mathrm{O} 1 / \mathrm{O} 2)$ as a betweensubjects factor confirmed that there was an effect of context, $F(1,14)=10.08, M S E=4.67, p<.01$, but no effect of devaluation, nor an interaction between these factors, $F_{\mathrm{S}}<1$. Similarly, we found a numerical tendency for the rate of magazine entries to be higher in rats sated with $\mathrm{O} 2$ rather than $\mathrm{O} 1$ during the first $3 \mathrm{~min}$ of the test (see Fig. 3). However, an ANOVA with Devaluation $(\mathrm{O} 1 / \mathrm{O} 2)$ and Minute as factors showed no effect of devaluation, $F(4,56)=1.46, M S E=$ $19.04, p>.23$, or trial, $F(4,56)=1.03, M S E=19.04$, $p>.40$, and no interaction between these factors, $F(1,14)=$ $1.25, M S E=46.21, p>.28$.

\section{Discussion}

The results of Experiment 1 are theoretically noteworthy, because they established that an instrumental renewal effect can be observed under circumstances in which the Pavlovian properties of the contexts (A and B) were equated, and served to establish the generality of the results recently reported by Todd (in press). In our case, unlike in Todd's experiments, the effect was seen following relatively little extinction (120 extinction trials on each lever) that ended approximately $1 \mathrm{~h}$ before the test. The contextual control exerted by the extinction context, in our case, was clearly be acquired very rapidly. ${ }^{1}$ The fact that this renewal effect was not influenced by whether rats were sated on the training reinforcer $(\mathrm{O} 1)$ or a novel reinforcer $(\mathrm{O} 2)$ prior to the test could be taken to suggest that

\footnotetext{
${ }^{1}$ In both Experiment 1 and in the experiments reported by Todd (in press), extinction of the Pavlovian properties of either the lever or the cues associated with the lever cannot be determined, which remains a potential source of the renewed responding that is observed.
} 


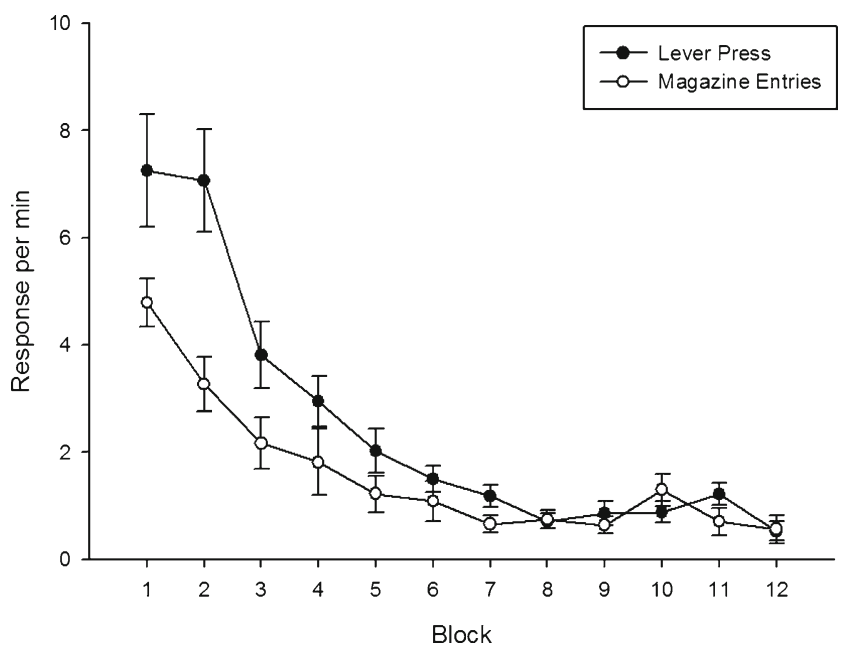

Fig. 1 Experiment 1, extinction: mean rates $(+S E M s)$ of lever pressing (with R1 and R2 pooled) and magazine entries (during periods when the levers were retracted) in responses per minute, over the course of the extinction sessions

renewed responding was insensitive to the current value of the outcome, and, in this sense, was not goal-directed but instead was habitual. This conclusion would be premature, however. There was a tendency for sating rats with the training outcome to reduce test responding and magazine entries, relative to sating them with the novel reinforcer. Also, the use of an experimental design in which the contexts were equated in terms of their Pavlovian properties required that they have the same relationship with a single outcome. This arrangement is not necessarily an optimal procedure for observing a selective satiation effect on instrumental performance. For example, the training procedure might have resulted in the rats not encoding

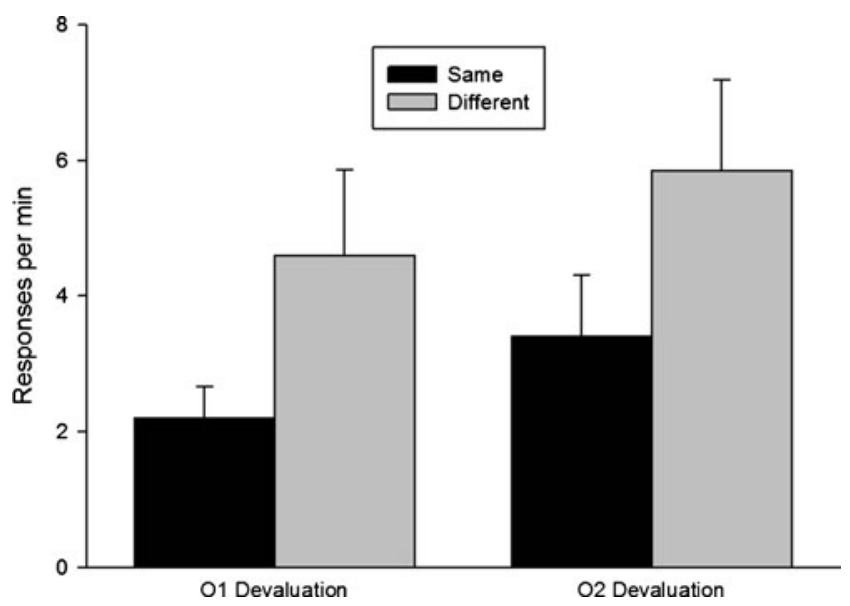

Fig. 2 Experiment 1, test: mean rates of instrumental responding (in responses per minute $+S E M \mathrm{~s}$ ) as a function of whether responding was tested in the same context as extinction (i.e., R1 in B and R2 in A) or in a different context (i.e., R1 in A or R2 in B). The rats had been sated either on the outcome associated with R1 and R2 during training $(\mathrm{O} 1)$ or on a novel outcome $(\mathrm{O} 2)$

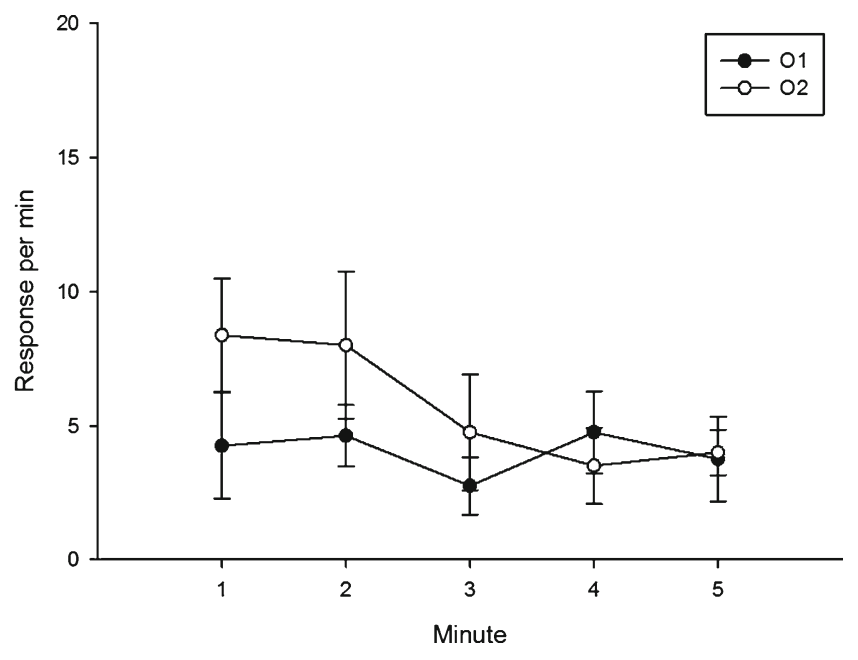

Fig. 3 Experiment 1, test: mean rates of magazine entries (in responses per minute $+S E M \mathrm{~s}$ ) over the 5-min extinction test, as a function of whether the rats had been sated on the outcome associated with R1 and $\mathrm{R} 2$ during training $(\mathrm{O} 1)$ or on a novel outcome $(\mathrm{O} 2)$

the sensory properties of the outcome, and, as we have mentioned, the satiation procedure necessarily confounded training outcome identity with outcome familiarity. Moreover, the fact that two extinction sessions (one with each lever) and a satiation session were experienced might have meant that any effects of satiation were difficult to detect. Given these considerations, in Experiment 2 we used a design in which the rats received training in which two responses (R1 and R2) were paired with different outcomes $(\mathrm{O} 1$ and $\mathrm{O} 2)$ in context $\mathrm{A}$, and then received extinction and were sated on $\mathrm{O} 1$ in context $\mathrm{B}$, prior to a test in either context A or B. This design closely resembled the instrumental renewal procedure used by Bouton et al. (2011), which was itself modeled on many of those used in studies of renewal in Pavlovian conditioning. This design would not equate the Pavlovian properties of contexts A and B in the same way as in Experiment 1. To do so would have required the use of four responses in conjunction with the two reinforcers - that is, with each member of a pair of two responses being separately associated with the two reinforcers in context $\mathrm{A}$, and the remaining pair being associated with the two reinforcers in context $\mathrm{B}$. However, the inclusion of further controls should help to establish the generality of the principal conclusion from Experiment 1: namely, that a renewal of instrumental responding could be observed that was not the consequence of the Pavlovian properties of the contexts.

\section{Experiment 2}

The design of Experiment 2 is summarized in the lower panel of Table 1. In this experiment, two instrumental lever-press responses (R1 and R2) were paired with different outcomes 
(O1 and O2) in context A. After training, the rats in Group Extinction were placed in context $\mathrm{B}$ and received presentations of one of the training outcomes (O1), followed by the opportunity to respond on both levers (R1 and R2). Group No Extinction simply received presentations of one of the training reinforcers $(\mathrm{O} 1)$. The use of a design in which both responses were extinguished in the same session as the rats were sated on one of the reinforcers (i.e., O1) allowed us to address some of the methodological concerns raised about Experiment 1. In particular, in Experiment 2 the rats were familiar with both outcomes, and combining the satiation procedure with the extinction procedure in a single session reduced the interval between the two operations and the critical test. One potential problem with combining these procedures, however, was that it might result in a form of response reduction that was not the product of conventional extinction. For example, it might result in a reduction in responding that was due to contingency degradation. However, monitoring the rates of R1 and R2 during extinction, in addition to magazine entries, allowed us to assess such explanations of any reduction in responding. For example, a reduction in responding due to contingency degradation should be more apparent in the case of R1 than of R2.

Rats then received a 5-min extinction test in either context A or B, with both levers present. For rats in Group Extinction, responding should be more evident in context A than in context $\mathrm{B}$, and if the renewed responding were goal-directed, then the level of R2 (which was paired with the outcome that remained valued) should exceed the level of R1 (which was paired with the now-devalued outcome). For the rats in Group No Extinction, the tests in contexts A and B would provide an assessment of the extent to which instrumental conditioning was context-specific and allow us to determine whether responding was goal-directed in the absence of any extinction treatment (cf. Adams, 1982; Adams $\&$ Dickinson, 1981). It would also allow us to gauge whether contexts A and B were having their effects on instrumental responding by virtue of differences in the Pavlovian properties of contexts A and B. If the contexts exerted an effect of instrumental responding through a difference in their associative strengths, then in Group No Extinction the level of instrumental responding (R1 and R2) should be greater in context A than in context B.

\section{Method}

Subjects, apparatus, and procedure The 32 naïve male Lister hooded rats were from the same supplier and were maintained in the same way as in Experiment 1. The apparatus was the same that had been used in Experiment 1, with the exception that only eight chambers were used instead of 16. Half of these chambers were decorated with black-andwhite checked wallpaper, and the remainder were decorated with white wallpaper bearing black spots. The rats were first trained to retrieve food pellets and sucrose from the food well in two separate 30-min sessions, in the same manner as in Experiment 1. Rats then received 12 sessions of instrumental conditioning in context A over 6 days that were conducted at the same time of day for each rat. The two types of instrumental conditioning trials (i.e., $\mathrm{R} 1 \rightarrow \mathrm{O} 1$ and $\mathrm{R} 2 \rightarrow \mathrm{O} 2$ ) occurred in alternating sessions that were presented in a counterbalanced order. In these sessions, one lever was inserted, and pressing this lever (e.g., R1, pressing the left lever) resulted in the delivery of the designated outcome (O1). In the first session of each response $\rightarrow$ outcome combination, reinforcement was delivered according to a CRF schedule, and on the remaining sessions, an RI 30-s schedule was employed. The rate of lever pressing (in rpm) was used to assess instrumental conditioning. The other details of the training stage were identical to those of Experiment 1.

On the final day, rats were randomly assigned to two groups: Extinction and No Extinction. The rats in Group Extinction were placed in context B and received 120 presentations of $\mathrm{O} 1$ (designed to sate them with this outcome) and 120 opportunities to respond on both levers. To be more specific, the delivery of $\mathrm{O} 1$ on an RT 30-s schedule was followed, once the rat had removed its snout from the food well, by the insertion of both levers for $10 \mathrm{~s}$. Responses on either lever had no programmed consequences. After this 10-s period, the levers were withdrawn until the next trial. Rats in Group No Extinction were also placed in context B and received exactly the same procedure, with the exception that the levers were not inserted into the chamber. Approximately 5 min after these sessions, the rats received a single 5-min test session in either context A or context B. Both levers were present, and no reinforcers were delivered. The other details of the test were the same as in Experiment 1.

\section{Results and discussion}

As in Experiment 1, lever pressing increased from the first RI 30 -s session (overall mean $=0.83 \mathrm{rpm}$; Group Extinction mean $=0.80 \mathrm{rpm}$, and Group No Extinction mean $=0.86 \mathrm{rpm}$ ) to the final, sixth day of training (overall mean $=7.61 \mathrm{rpm}$; Group Extinction mean $=7.83 \mathrm{rpm}$, and Group No Extinction mean $=7.39 \mathrm{rpm}$ ). An ANOVA with Group and Day as factors revealed no effect of group, $F<1$, an effect of day, $F(5,150)=67.99, M S E=3.77, p<.001$, and no interaction between these two factors, $F<1$. Inspection of Fig. 4 reveals a marked reduction in lever pressing across the extinction session for Group Extinction, but little difference in the rates of R1 and R2. This description of the results was confirmed by an ANOVA that revealed no significant main effect of response (R1 vs. R2), $F<1$, a main effect of block, $F(11,165)=13.56$, $M S E=1.8, p<.001$, and no interaction between these factors, $F<1$. The fact that the rates of $\mathrm{R} 1$ and $\mathrm{R} 2$ declined at the same 


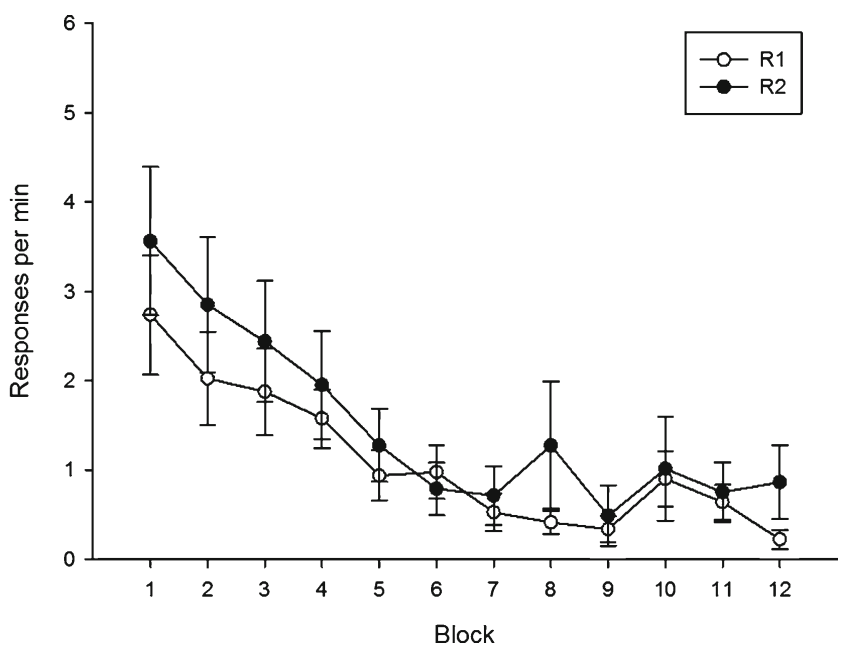

Fig. 4 Experiment 2, extinction of lever pressing: mean rates of instrumental responding (in responses per minute $+S E M \mathrm{~s}$ ) during extinction for Group Extinction

rates suggests that the presentation of $\mathrm{O} 1$ was not influencing performance through an outcome-specific contingency degradation effect, which should have resulted in the rate of R1 being lower than that of R2 (e.g., Colwill \& Rescorla, 1986). Inspection of Fig. 5 shows that in both groups the rates of magazine entry also declined over the course of the extinction session (note that levers were retracted during this period for Group Extinction). ANOVA confirmed that there was no significant main effect of group, $F<1$, a main effect of block, $F(11,165)=9.31, M S E=7.72, p<.001$, and no interaction between these factors, $F<1$.

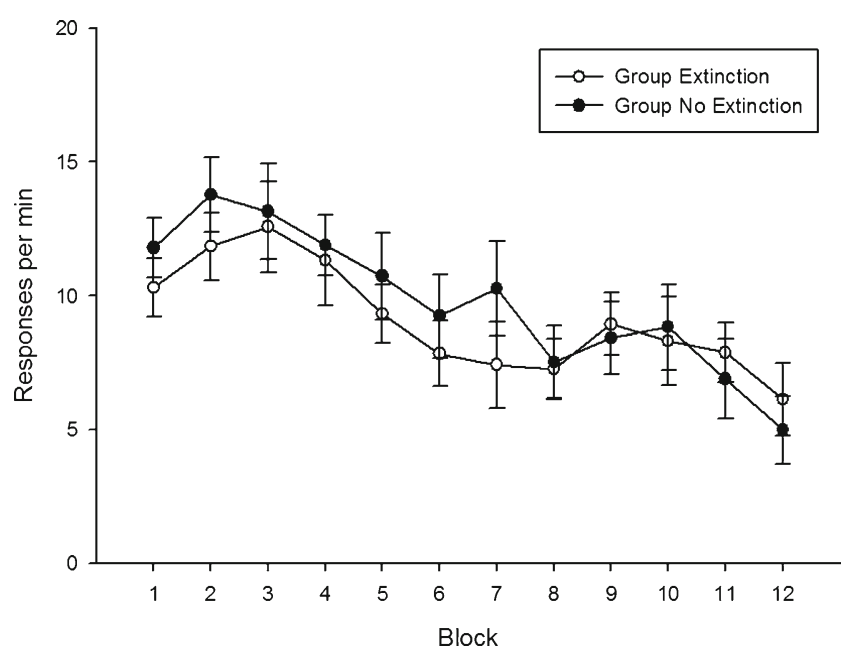

Fig. 5 Experiment 2, extinction of magazine entries: mean rates of magazine entry (in responses per minute $+S E M$ s) for Groups Extinction and No Extinction. The rats in both groups were sated with $\mathrm{O} 2$ prior to the test, and whereas rats in Group Extinction received instrumental extinction in context B, those in Group No Extinction did not. Magazine entries for Group Extinction are shown for the periods when the levers were retracted
The results of principal interest, from the test of Experiment 2, are summarized in Fig. 6. This figure reveals that there was more responding in context A than in context B for Group Extinction, and that this renewed responding in $\mathrm{A}$ (and the extinguished responding in B) was sensitive to the current value of the outcome. That is, the rats in Group Extinction were more likely to perform R2 than R1 in both contexts A and B. In contrast, in Group No Extinction the context in which testing occurred (A or B) had no effect on the level of responding (cf. Bouton et al., 2011), but sating rats on $\mathrm{O} 1$ resulted in them being more likely to perform R2 than R1. This pattern of results suggests that, using our procedures, rats readily identified the levers that supported $R 1$ and $R 2$ in context $\mathrm{B}$, and that the abilities of $\mathrm{R} 1$ and $\mathrm{R} 2$ to retrieve their respective outcomes, $\mathrm{O} 1$ and $\mathrm{O} 2$, were unaffected by being placed in context B. An ANOVA with Response (R1 or R2) as a within-subjects factor and Context (A or B) and Group (Extinction or No Extinction) as between-subjects factors revealed effects of response, $F(1,28)=6.01, M S E=4.08$, $p<.05$, group, $F(1,28)=9.52, p<.005$, and context, $F(1,28)=$ $6.04, M S E=3.064, p<.05$. We also observed an interaction between group and context, $F(1,28)=4.590, M S E=3.064$, $p<.05$, but no other interactions, $F_{\mathrm{S}}<1$. To analyze the nature of this interaction, separate ANOVAs were conducted on the results from Groups No Extinction and Extinction. The analysis for Group Extinction revealed main effects of context, $F(1,14)=$ 11.22, $M S E=2.43, p<.005$, and response, $F(1,14)=5.46$, $M S E=2.89, p<.05$, but no interaction between these factors, $F<1$. The equivalent analysis for Group No Extinction revealed no effect of context, $F<1$, no significant effect of

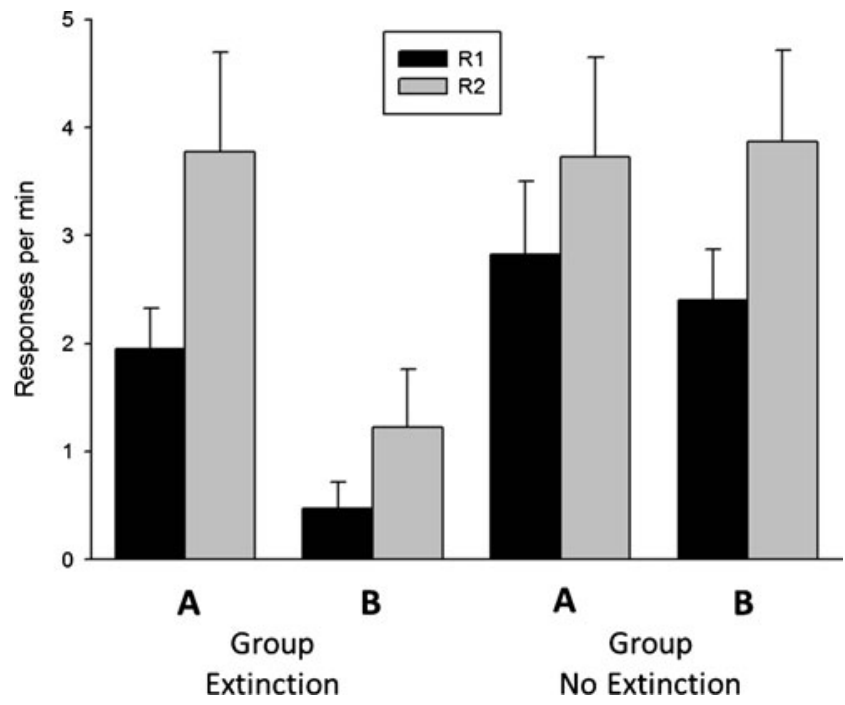

Fig. 6 Experiment 2, test: mean rates of two responses (R1 and R2, in responses per minute $+S E M \mathrm{~s}$ ) as a function of whether testing was conducted in the conditioning context (A) or the extinction context (B). Group Extinction received extinction with both responses (R1 and R2) and were sated with the outcome associated with R1 (i.e., O1) in context B, and Group No Extinction were simply sated with O1 in context B 
response, $F(1,14)=1.97, M S E=5.73, p>.18$, and no interaction between these factors, $F<1$. Although the inclusion of Response as a factor in these supplementary analyses was not licensed by the results of the omnibus analysis (in which the overall effect of response did not interact with the other factors), its inclusion certainly established the critical point that there was a significant effect of response (R1 or R2) occurred in Group Extinction: The analysis thereby that responding observed after extinction was goal-directed, whether this responding was subject to a process of renewal (in context A) or not (in context B).

Inspection of Fig. 7 shows that the overall rates of magazine entries were somewhat lower in Group Extinction (upper panel) than in Group No Extinction (lower panel). It also reveals that although the rate of magazine entries was
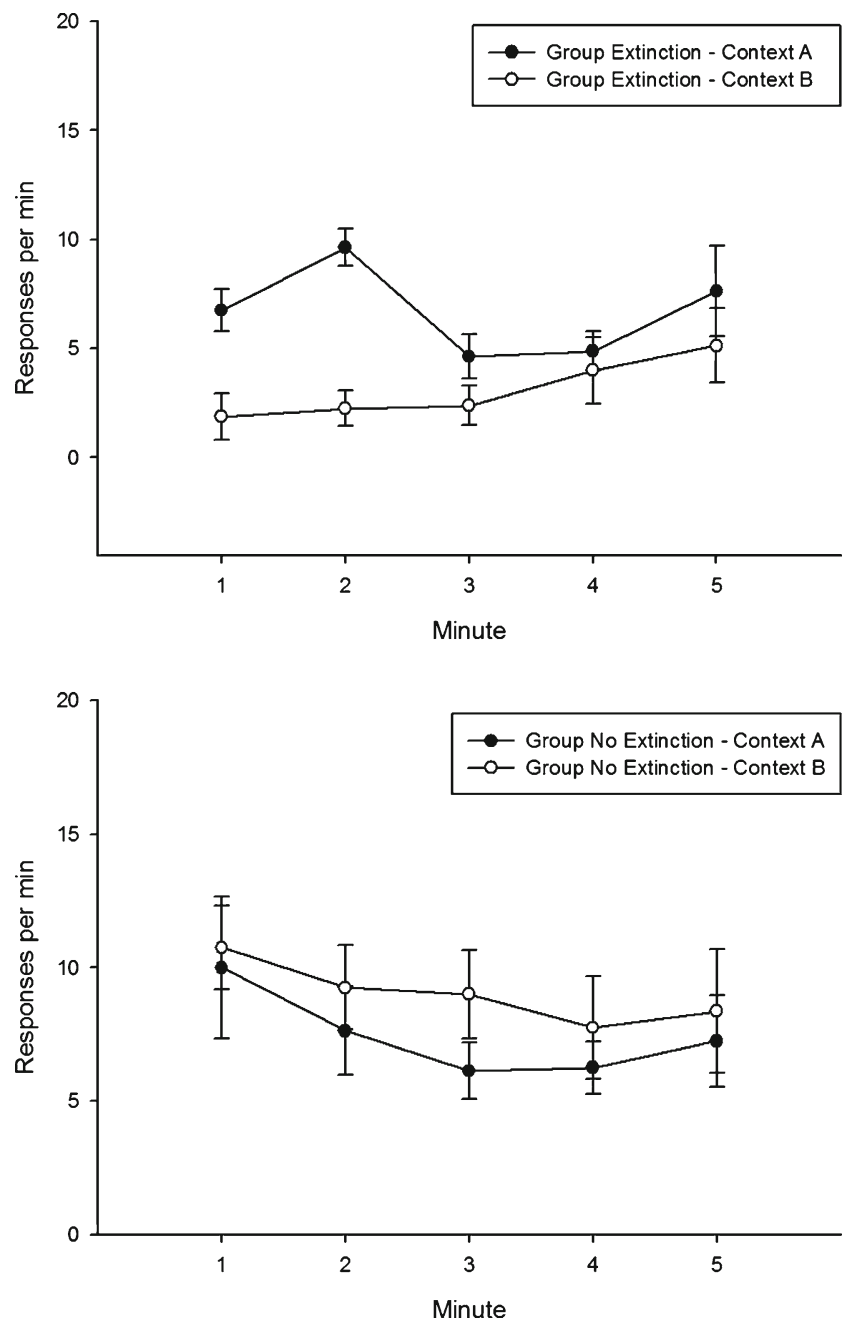

Fig. 7 Experiment 2, test: mean rates of magazine entries (in responses per minute $+S E M$ s) over the 5-min extinction test, as a function of whether rats were tested in context A or B. Group Extinction (upper panel) received extinction of both instrumental responses (R1 and R2) and were sated with the outcome associated with R1 (i.e., O1) in context B, and Group No Extinction were simply sated with $\mathrm{O} 1$ in context B higher in context A than in context B for Group Extinction, this was not the case for Group No Extinction. An ANOVA with Minute, Group, and Context as factors confirmed the findings of an effect of group, $F(1,28)=11.23, M S E=39.39$, $p<.005$, no effect of minute, $F(4,112)=1.77, M S E=13.84$, $p>.14$, and no interaction between these factors, $F(4,112)=$ $1.86, M S E=13.84, p>.12$. Also, although we found no main effect of context, $F(1,28)=1.02, M S E=39.39, p>.32$, or interaction between minute and context, $F(4,112)=1.18$, $M S E=13.84, p>.32$, as well as no three-way interaction $(F<1)$, a group by context interaction was found, $F(1,28)=$ $6.73, p<.05$. Analysis of this interaction showed that whereas there was more responding in context $\mathrm{A}$ than in context $\mathrm{B}$ for Group Extinction, $t(14)=3.09, p<.01$, no difference in responding was apparent between contexts $\mathrm{A}$ and $\mathrm{B}$ in Group No Extinction, $t(14)=-0.977, p>.35$. This pattern of results mirrored the overall differences in lever-press responding among the four groups, in the sense that in the rats given extinction, more lever pressing was evident in context $\mathrm{A}$ than in context $\mathrm{B}$, whereas this was not the case in the rats that had not received extinction. The relationship between the overall levels of magazine entries and the overall levels of lever pressing is difficult to disentangle, but the nature of this relationship does not undermine the principal conclusion that one can draw from Experiment 2: namely, that renewed lever pressing is as sensitive to outcome devaluation as is lever pressing that has not been subject to an extinction treatment.

\section{General discussion}

The processes of conditioning and extinction are fundamental to any understanding of learned behavior (Hull, 1943; Spence, 1937). One of the key features of Pavlovian conditioning and extinction is the relative lability of extinction in the face of changes in the context in which it is assessed. For example, in Pavlovian conditioning procedures, a change in context that has little or no apparent effect (cf. Hall \& Honey, 1990) on the level of responding generated by reinforced training will markedly attenuate the influence of extinction on performance (see Bouton, 2004). This feature of Pavlovian conditioning is also true of instrumental conditioning. For example, if rats receive instrumental conditioning in context $\mathrm{A}$ and are then given extinction in context $\mathrm{B}$, placement in context $\mathrm{A}$ results in renewed instrumental responding (e.g., Bouton et al., 2011; Todd et al., 2012). Here, we investigated the source of renewed instrumental responding in two experiments with rats. Experiment 1 showed that renewed instrumental conditioning can be observed without concomitant differences in the excitatory or inhibitory Pavlovian properties of the two contexts, A and B; and Experiment 2 showed that renewed instrumental responding was every bit as sensitive to the current value of the outcome as was instrumental responding 
that had not been subject to an extended period of extinction. These observations begin to allow a more secure interpretation to be given to ABA renewal in an instrumental setting, and this interpretation also has implications for other domains of research.

The fact that ABA instrumental renewal can be observed when the Pavlovian relationship between the contexts, A and $\mathrm{B}$, and reinforcement and nonreinforcement have been equated leaves open two obvious potential loci for the context in which extinction occurs to exert its influence on instrumental performance. One resides in the capacity for the context to directly affect the response program responsible for instrumental behavior. The extinction of instrumental behavior might result in the extinction context (i.e., B) coming to reduce the likelihood that the motor program for the extinguished response will become active. For example, it has been argued that a stimulus that has undergone extinction might come to inhibit the response that was extinguished in its presence (Rescorla, 1993), and the same might be true of a context in which an instrumental response has been extinguished. Application of this suggestion to the results of Experiment 1 is straightforward: After extinction training involving R2 in context A and R1 in context B, context A might come to inhibit R2, and context B might inhibit R1. Under these conditions, R1 would be released from this inhibitory influence when tested in context $\mathrm{A}$, and R2 would be released from this influence when assessed in context B. Of course, this analysis would still leave open the possibility that the current value of the reinforcer would interact with renewed responding (see Exp. 2), to the extent that instrumental behavior was also controlled by response $\rightarrow$ outcome associations.

The second locus is less direct. According to this alternative, the extinction context (e.g., B) comes to gate and augment the inhibitory $\mathrm{R} 1 \rightarrow \mathrm{O} 1$ association, and placing rats in context A removes this gating influence and allows the excitatory $\mathrm{R} 1 \rightarrow \mathrm{O} 1$ association to be more evident (Bouton et al., 2011). Again, the influence of this excitatory association will be constrained by the current value of O1. Several features of the results of Experiments 1 and 2 are consistent with this form of hierarchical control of inhibitory associations. In Experiment 1, any ability of contexts $\mathrm{A}$ and $\mathrm{B}$ to gate the excitatory links between R1 and $\mathrm{O} 1$ and R2 and O1 (e.g., Hall \& Honey, 1989, 1990; Honey, Willis, \& Hall, 1990; for a modified configural analysis, see Honey \& Watt, 1999) was necessarily equated, leaving the inhibitory $\mathrm{R} 1 \rightarrow \mathrm{O} 1$ and $\mathrm{R} 2 \rightarrow \mathrm{O} 1$ links acquired during extinction as the sole target for hierarchical contextual control. Moreover, in Experiment 2 we found no effect of a change of context on instrumental conditioning that had not been subject to prolonged extinction (cf. Bouton et al., 2011). The fact that instrumental behavior was equally evident in contexts $\mathrm{A}$ and $\mathrm{B}$ in rats that had not received extinction did not appear to reflect a ceiling effect or some general insensitivity of our response measure: We observed no effect of a change in context on R1 (whose outcome had been devalued) or on R2 (whose outcome was not devalued). Irrespective of the precise locus of contextual control, the results of Experiments 1 and 2 inform two other domains of research: clinical and neuroscientific.

It has been argued that where forms of human psychopathology involve conditioning - most notably, drug dependence, anxiety disorders, and overeating - extinction treatments might be clinically applied to eliminate distressing thoughts and behaviors (Bouton, 2002, 2011; Conklin \& Tiffany, 2002; Craske et al., 2008; Myers \& Davis, 2002; Thomas, Larsen, \& Ayres, 2003). The findings of Experiments 1 and 2 reinforce the general view that the efficacy of such treatments might be constrained by the fact that extinction is context-specific - with relapse being the consequence of a failure of extinction to generalize to new environments. However, our results also suggest that these failures to generalize do not necessarily reflect any simple relationship between the extinction context (or the test context) and reinforcing events that have occurred in those contexts. Rather, they suggest that the extinction context has a more specific role - either setting the occasion for the inhibitory relationship between responses (e.g., drug seeking) and outcomes (drug consumption) or directly inhibiting the response. Behavioral training regimens that augment either of these processes are clear targets for future basic and applied research. The results of Experiment 2 reveal that renewed responses are sensitive to the current value of the outcome. The applied significance of this finding is straightforward: For example, renewed drug seeking during relapse is likely to be directed toward the drug (or its effects) that motivated the original drug-seeking behavior prior to any extinction treatment. The implication of this view is that behavioral treatment strategies will stand a greater chance of success if they are combined with other medical treatments that decrease the motivational value of the drug (Volkow \& Li, 2004).

The prelimbic region (PL) of the medial prefrontal cortex has been directly implicated in ABA renewal. Thus, inactivation of the PL, at test, attenuates ABA renewal of extinguished alcohol-seeking behavior (e.g., lever pressing; Willcocks \& McNally, 2012; see also Hamlin, Newby, \& McNally, 2007; Zironi, Burattini, Aicardi, \& Janak, 2006; for a review, see Van den Oever, Spijker, Smit, \& De Vries, 2010). The results of Experiments 1 and 2 established that, at least for the case of instrumental conditioning with food, the extinction context (B) modulates the impact of response $\rightarrow$ outcome associations on behavior either by directly inhibiting the response or by gating the inhibitory response $\rightarrow$ outcome association. That is, the results provide support for the view that there is a goal-directed component to renewed instrumental responding (a seeking component) and that the context modulates this component. An attenuation of ABA renewal observed after inactivation of the PL, for example, might then reflect a disruption to a 
contextual inhibitory process or to the interaction between this process and the response $\rightarrow$ outcome association. However, identifying the specific roles of brain structures in the renewal of extinguished responding awaits the use of procedures that discriminate between the differing ways in which a renewal effect could be generated.

To conclude: We have shown that renewed instrumental conditioning can be observed without concomitant involvement of any excitatory or inhibitory Pavlovian properties of the contexts, and that renewed responding is just as sensitive to the current value of the outcome as is instrumental responding that has not been subject to an extended period of extinction. Taken together, these results suggest that the extinction context exerts an indirect or a direct inhibitory influence on the effectiveness of the instrumental response $\rightarrow$ outcome association, the removal of which allows the impact of the response $\rightarrow$ outcome association on performance to be revealed.

Author Note These results were supported by a studentship awarded to S.R.C-H. by the School of Psychology.

\section{References}

Adams, C. D. (1982). Variations in the sensitivity of instrumental responding to reinforcer devaluation. Quarterly Journal of Experimental Psychology, 34B, 77-98.

Adams, C. D., \& Dickinson, A. (1981). Instrumental responding following reinforcer devaluation. Quarterly Journal of Experimental Psychology, 33B, 109-121.

Baker, A. G., Steinwald, H., \& Bouton, M. E. (1991). Contextual conditioning and reinstatement of extinguished instrumental responding. Quarterly Journal of Experimental Psychology, 43B, 199-218.

Bossert, J. M., Liu, S. Y., Lu, L., \& Shaham, Y. (2004). A role of ventral tegmental area glutamate in contextual cue-induced relapse to heroin seeking. Journal of Neuroscience, 24, 10726-10730.

Bouton, M. E. (2002). Context, ambiguity, and unlearning: Sources of relapse after behavioral extinction. Biological Psychology, 52, 976-986. doi:10.1016/S0006-3223(02)01546-9

Bouton, M. E. (2004). Context and behavioral processes in extinction. Learning and Memory, 11, 485-494. doi:10.1101/lm.78804

Bouton, M. E. (2011). Learning and the persistence of appetite: Extinction and the motivation to eat and overeat. Physiology \& Behavior, 103, 51-58.

Bouton, M. E. (2012). Relapse processes after the extinction of instrumental learning: Renewal, resurgence, and reacquisition. Behavioural Processes, 90, 130-141.

Bouton, M. E., \& Bolles, R. C. (1979). Contextual control of the extinction of conditioned fear. Learning and Motivation, 10, 445-466.

Bouton, M. E., \& King, D. A. (1983). Contextual control of the extinction of conditioned fear: Tests for the associative value of the context. Journal of Experimental Psychology. Animal Behavior Processes, 9, 248-265. doi:10.1037/0097-7403.9.3.248

Bouton, M. E., \& Swartzentruber, D. (1986). Analysis of the associative and occasion setting properties of contexts participating in a Pavlovian discrimination. Journal of Experimental Psychology.
Animal Behavior Processes, 12, 333-350. doi:10.1037/00977403.12.4.333

Bouton, M. E., Todd, T. P., Vurbic, D., \& Winterbauer, N. E. (2011). Renewal after the extinction of free-operant behavior. Learning \& Behavior, 39, 57-67. doi:10.3758/s13420-011-0018-6

Bouton, M. E., Winterbauer, N. E., \& Todd, T. P. (2012). Relapse processes after the extinction of instrumental learning: Renewal, resurgence, and reacquisition. Behavioral Processes, 90, 130-141. doi:10.1016/j.beproc.2012.03.004

Cohen-Hatton, S. R., Haddon, J. E., George, D. N., \& Honey, R. C. (2013). Pavlovian-to-instrumental transfer: Paradoxical effects of the Pavlovian relationship explained. Journal of Experimental Psychology. Animal Behavior Processes, 39, 14-23.

Colwill, R. M., \& Rescorla, R. A. (1986). Associative structures in instrumental learning. In G. H. Bower (Ed.), The psychology of learning and motivation (Vol. 20, pp. 55-104). Orlando, FL: Academic Press.

Colwill, R. M., \& Rescorla, R. A. (1988). Associations between the discriminative stimulus and the reinforcer in instrumental learning. Journal of Experimental Psychology. Animal Behavior Processes, 14, 155-164.

Conklin, C. A., \& Tiffany, S. T. (2002). Applying extinction research and theory to cue-exposure addiction treatments. Addiction, 97, $155-167$.

Craske, M. G., Kircanski, K., Zelikowsky, M., Mystkowski, J., Chowdhury, N., \& Baker, A. (2008). Optimizing inhibitory learning during exposure therapy. Behaviour Research and Therapy, 46, 5-27.

Crombag, H. S., \& Shaham, Y. (2002). Renewal of drug seeking by contextual cues after prolonged extinction in rats. Behavioral Neuroscience, 116, 169-173.

de Wit, S., \& Dickinson, A. (2009). Associative theories of goal-directed behaviour: A case for animal-human translational models. Psychological Research, 73, 463-476.

Delamater, A. R., LoLordo, V., \& Sosa, W. (2003). Outcome-specific conditioned inhibition in Pavlovian backward conditioning. Learning and Behaviour, 31, 393-402.

Hall, G., \& Honey, R. C. (1989). Contextual effects in conditioning, latent inhibition, and habituation: Associative and retrieval functions of contextual cues. Journal of Experimental Psychology. Animal Behavior Processes, 15, 232-241. doi:10.1037/00977403.15.3.232

Hall, G., \& Honey, R. C. (1990). Context-specific conditioning in the conditioned-emotional-response procedure. Journal of Experimental Psychology. Animal Behavior Processes, 16, 271-278. doi:10.1037/ 0097-7403.16.3.271

Hamlin, A. S., Clemens, K. J., \& McNally, G. P. (2008). Renewal of extinguished cocaine-seeking. Neuroscience, 151, 659-670.

Hamlin, A. S., Newby, J., \& McNally, G. P. (2007). The neural correlates and role of D1 dopamine receptors in renewal of extinguished alcohol-seeking. Neuroscience, 146, 525-536.

Harris, J. A., Jones, M. L., Bailey, G. K., \& Westbrook, R. F. (2000). Contextual control over conditioned responding in an extinction paradigm. Journal of Experimental Psychology. Animal Behavior Processes, 26, 174-185.

Holmes, N. M., Marchand, A. R., \& Coutureau, E. (2010). Pavlovian to instrumental transfer: A neurobehavioral perspective. Neuroscience and Biobehavioral Reviews, 34, 1277-1295.

Honey, R. C., \& Watt, A. (1999). Acquired relational equivalence between contexts and features. Journal of Experimental Psychology. Animal Behavior Processes, 25, 324-333.

Honey, R. C., Willis, A., \& Hall, G. (1990). Context specificity in pigeon autoshaping. Learning and Motivation, 21, 125-136.

Hull, C. L. (1943). Principles of behavior: An introduction to behavior theory. New York, NY: Appleton Century Crofts.

Laborda, M. A., McConnell, B. L., \& Miller, R. R. (2011). Behavioral techniques to reduce relapse after exposure therapy: Applications 
of studies of experimental extinction. In T. R. Schachtman \& S. Reilly (Eds.), Associative learning and conditioning theory: Human and non-human applications (pp. 79-103). Oxford, UK: Oxford University Press.

Myers, K. M., \& Davis, M. (2002). Behavioral and neural analysis of extinction. Neuron, 36, 567-584.

Nakajima, S., Tanaka, S., Urushihara, K., \& Imada, H. (2000). Renewal of extinguished lever-press responses upon return to the training context. Learning and Motivation, 31, 416-431.

Nakajima, S., Urushihara, K., \& Masaki, T. (2002). Renewal of operant performance formerly eliminated by omission or non-contingency training upon return to the acquisition context. Learning and Motivation, 33, 510-525.

Pearce, J. M., \& Hall, G. (1979). The influence of context-reinforcer associations on instrumental performance. Animal Learning \& Behavior, 7, 504-508.

Rescorla, R. A. (1991). Associative relations in instrumental learning: The eighteenth Bartlett memorial lecture. Quarterly Journal of Experimental Psychology, 43B, 1-23.

Rescorla, R. A. (1993). Inhibitory associations between S and R in extinction. Animal Learning \& Behavior, 21, 327-336.

Rescorla, R. A. (2008). Within-subject renewal in sign tracking. Quarterly Journal of Experimental Psychology, 61, 1793-1802. doi: $10.1080 / 17470210701790099$

Rescorla, R. A., \& Solomon, R. L. (1967). Two-process learning theory: Relationships between Pavlovian conditioning and instrumental learning. Psychological Review, 74, 151-182. doi:10.1037/h0024475
Skinner, B. F. (1938). The behavior of organisms. New York, NY: Appleton-Century-Crofts.

Spence, K. W. (1937). The differential response in animals to stimuli varying within a single dimension. Psychological Review, 44, 430-444.

Thomas, B. L., Larsen, N., \& Ayres, J. J. B. (2003). Role of context similarity in $\mathrm{ABA}, \mathrm{ABC}$, and $\mathrm{AAB}$ renewal paradigms: Implications for theories of renewal and for treating human phobias. Learning and Motivation, 34, 410-436.

Todd, T. P. (in press). Mechanisms of renewal after the extinction of instrumental behavior. Journal of Experimental Psychology: Animal Behavior Processes.

Todd, T. P., Winterbauer, N. E., \& Bouton, M. E. (2012). Effects of amount of acquisition and contextual generalization on the renewal of instrumental behavior after extinction. Learning \& Behavior, 40, $145-157$.

Van den Oever, M. C., Spijker, S., Smit, A. B., \& De Vries, T. J. (2010). Prefrontal cortex plasticity mechanisms in drug seeking and relapse. Neuroscience and Biobehavioral Reviews, 35, 276-284.

Volkow, N. D., \& Li, T. K. (2004). Drug addiction: The neurobiology of behaviour gone awry. Nature Reviews Neuroscience, 5, 963-970.

Willcocks, A. L., \& McNally, G. P. (2012). The role of medial prefrontal cortex in extinction and reinstatement of alcohol-seeking in rats. European Journal of Neuroscience, 37, 259-268.

Zironi, I., Burattini, C., Aicardi, G., \& Janak, P. H. (2006). Context is a trigger for relapse to alcohol. Behavioural Brain Research, 167, $150-155$. 